Revue d'histoire de l'Amérique française

DEYUE D.HISTOIRE DE L'AMÉRIQUE FRANÇAISE

\title{
Le dossier biographique de Jean Prat
}

\section{Roland Lamontagne}

Volume 16, numéro 2, septembre 1962

URI : https://id.erudit.org/iderudit/302195ar

DOI : https://doi.org/10.7202/302195ar

Aller au sommaire du numéro

Éditeur(s)

Institut d'histoire de l'Amérique française

ISSN

0035-2357 (imprimé)

1492-1383 (numérique)

Découvrir la revue

Citer cet article

Lamontagne, R. (1962). Le dossier biographique de Jean Prat. Revue d'histoire de l'Amérique française, 16(2), 219-224. https://doi.org/10.7202/302195ar d'utilisation que vous pouvez consulter en ligne.

https://apropos.erudit.org/fr/usagers/politique-dutilisation/ 


\section{LE DOSSIER BIOGRAPHIQUE DE JEAN PRAT *}

Les documents émanant de Jean Prat, ou le concernant, sont relativement peu nombreux. Outre la correspondance au nombre de vingt-huit lettres dont vingt-quatre, adressées de la NouvelleOrléans, s'échelonnent depuis 1735 jusqu'en 1746, le dossier de Jean Prat contient des pièces cotées S.56 et S.26 provenant des archives de l'Université de Montpellier. Le ministre Maurepas avait envoyé deux dépêches à Jean Prat: un mémoire d'information sur l'arbre à cire ${ }^{1}$ et une lettre de félicitation. ${ }^{2}$ Les microfilms des registres du Conseil supérieur de la Louisiane, au nombre de vingt-sept bobines pour la période de 1724 à 1746, constituent une source de renseignements relatifs à l'œuvre des frères Louis et Jean Prat en Louisiane. ${ }^{3}$ Jean F. Leroy a fait allusion à la correspondance Prat-Jussieu. ${ }^{4}$ Mettant à profit les travaux de John Duffy, ${ }^{5}$ le professeur Ewan a signalé, dans un

\footnotetext{
* Nous exprimons notre vive gratitude à Mlle Y. Vidal, conservateur honoraire des Archives de la Faculté de Médecine de l'Université de Montpellier, qui nous a communiqué une copie dactylographiée des dossiers académiques de Louis et de Jean Prat. Nous tenons à remercier M. Jean F. Leroy, Sous-Directeur au Muséum National d'Histoire naturelle, M. le Professeur Aubréville, Laboratoire de Phanérogamie au Muséum, et $\mathbf{M}$. Yves Laissus, Archiviste-Paléographe et Bibliothécaire au Muséum, d'avoir eu l'obligeance de nous transmettre un microfilm de la correspondance Prat-Jussieu. Monsieur Laissus nous a très obligeamment fait parvenir une copie exactement faite de la lettre, difficile à déchiffrer sur microfilm, libellée comme suit: A La Nelle Orléans, le 29e Juin 1740. Nous prions Mmes M. Densky, Bibliothécaire de l'Université de Montréal; C. G. Griffith, Director of the Manuscripts Division, Tulane University Library; et MM. P. Brunet, Archiviste national adjoint; J. Bousquet, Archiviste en Chef de l'Aveyron; L. Dulieu, Médecin-Commandant et historien des sciences; J. Ewan, Professeur à l'Université Tulane, qui nous ont fourni de précieuses indications, de bien vouloir trouver ici l'expression de notre reconnaissance.

1 Archives publiques du Canada, AC, B, 72: 484.

2 Ibid., AC, B, 65-4 : 1019.

3 Manuscripts Division, Tulane University Library. Cf. Louisiana Historical Quarterly, vol. 1-17.

4 Les botanistes français en Amérique du Nord avant 1850 (Paris, 1957), 285-286.

5 John Duffy, éd., The Rudolph Matas History of Medicine in Louisiana (Louisiana State Úniversity, 1958), 1:120-123 et passim.
} 
article sur Fougeroux de Bondaroy, ${ }^{6}$ l'apport de Jean Prat à la botanique.

Le nom de Prat est très répandu dans la montagne aveyronnaise. Selon Monsieur Jacques Bousquet, archiviste du Département de l'Aveyron et de l'ancien pays de Rouergue, la généalogie de la famille Prat n'a pas été étudiée. ${ }^{7}$

Les Archives de l'Université de Montpellier n'indiquent pas la date de naissance de l'étudiant; à l'immatriculation, celui-ci mentionnait le lieu d'où il venait, ce qui n'était pas forcément le lieu de naissance. Les registres matricules, à partir du XIX siècle, portent le lieu et la date de naissance de l'étudiant.

Louis Dulieu a analysé le mouvement scientifique montpelliérain au XVIII ${ }^{e}$ siècle ${ }^{8}$ et Hervé Harant a retracé l'histoire du Jardin des Plantes de Montpellier ; ${ }^{9}$ leurs recherches éclairent le milieu scientifique où se forma Jean Prat sous l'égide de François Chicoyneau. Professeur et chancelier de l'Université de Montpellier, François Chicoyneau (1672-1752) devint médecin de Louis XV à la mort de Pierre Chirac dont il était le gendre. Lors de la peste de 1720 , il avait dirigé la mission médicale à Marseille. Ce fut Roland-Michel Barrin de La Galissonière ${ }^{10}$ qui lui succéda à l'Académie des Sciences, en 1752.

Voici les renseignements que contiennent les Archives de l'Université de Montpellier au sujet de Jean Prat:

6 J. Ewan, « Fougeroux de Bondaroy (1732-1789) and His Projected Revision of Duhamel du Monceau's Traité (1755) on Trees and Shrubs. 1. An Analytical Guide to Persons, Gardens, and Works Mentioned in the Manuscripts 》, Proceedings of the American Philosophical Society, vol. 103 (1959), 807-818. -

Roland-Michel Barrin de La Galissonière et Pierre Bouguer avaient été nommés par l'Académie de Marine pour examiner cet ouvrage de Henri-Louis Duhamel du Monceau, Traité des arbres et arbustes qui se cultivent en France en pleine terre (2 vol., Paris, 1755). - Id., Ibid., 2: 386.

${ }^{7}$ Les registres paroissiaux de Laguiole (depuis 1659) existent à la mairie de cette localité.

8 Revue d'Histoire des Sciences et de leurs applications, $\mathrm{n}^{\circ} 3$ (1958), $\mathrm{XI}: 227-249$.

9 Endeavour, $\mathrm{n}^{\circ} 50$ (1954), XIII : 97-100.

$10 \mathrm{R}$. Lamontagne, «Chronologie de la carrière de La Galissonière 》, Revue d'Histoire des Sciences ..., nos 3-4 (1961), XIV : 255-256. 


\section{I. - REGISTRE S.56. ${ }^{11}$}

Mr. Jean Prat - guiolensis apud Ruthenos est passé Bachelier sous Mr. Chicoyneau Chancelier le 15 ème de Janvier 1731 ...

Mr. Prat a fait son $1^{\text {er }}$ examen sous Mr. Magnol ${ }^{12}$ le 17 avril 1731

son 2 ème examen sous Mr. Haguenot ${ }^{13}$ le 19 avril 1731

son 3 ème examen sous Mr. Chicoyneau père [François , 1672-1752]

le 22 avril 1731

son 4 ème examen sous Mr. Lazerme ${ }^{14}$ le 23 avril 1731

Mr. Prat a resceu sa licence de Mons. Levesque de Montpelier (sic) [Charles Joachim Colbert de Croissy] ${ }^{15}$ en présence de Mmrs Rideux ${ }^{16}$ et Magnol le 28 ème de may 1731

Mr. Prat a fait son Point Rigoureux ${ }^{17}$ le 20 ème de may 1731 [Les pages du registre sont inversées]

Mr. Prat a fait sa triduane ${ }^{18}$ lundy matin sous $\mathrm{Mr}$. Chicoyneau chancelier et le soir sous Mr. Rideux le 30 ème avril 1731

Mr. Prat a fait sa triduane mardy matin sous $\mathrm{Mr}$. Magnol et le soir sous Mr. Deidier ${ }^{19}$ le $1^{\text {er }}$ may 1731 $\mathrm{Mr}$. Prat a fait sa triduane mercredy matin sous $\mathrm{Mr}$. Haguenot et le soir sous Mr. Lazerme le 2 ème de may 1731

11 Registre des Actes commencé en 1706 et fini en 1732. Archives de l'Université de Montpellier.

12 Antoine Magnol qui avait succédé à son père, Pierre, à l'Université de Montpellier, ne s'est pas particulièrement distingué. Pierre Magnol (1638-1715), illustre botaniste, s'essaya le premier à classer les plantes par familles.

13 Jean-Henri Haguenot fut le premier titulaire de la chaire créée, en 1715, pour le service des pauvres. Son fils, Henri, le remplaça.

14 Jacques Lazerme, professeur; personnage d'importance secondaire.

15 Voir L. Dulieu, Revue d'Histoire des Sciences..., (1958), XI : 232.

16 Pierre Rideux, professeur; personnage d'importance secondaire.

17 Le cinquième diplôme, ou du Point Rigoureux, résume le curriculum des épreuves subies par le bachelier en vue de la licence.

18 Triduanes: épreuves subies trois jours durant, le matin et le soir.

19 Antoine Deidier (1670-1746), professeur de chimie. Membre de la mission médicale à Marseille, lors de la peste de 1720 , il exprima des vues exactes sur la cause de cette maladie. René Taton, éd., La Science moderne. Collection d'histoire générale des sciences (Paris, 1958), 2: 633. 
Mr. Jean Prat guiolensis apud Ruthenos est passé docteur sous Mr. Magnol le 4 ème de juin 1731 .

\section{II. - REGISTRE S.26. ${ }^{20}$}

Jean prat : Ego Joannes Prat guiolensis apud Ruthenos primum profiteor apud acta universitatis medicina monspeliensis die vigesima nona augusti anni 1728

$$
\text { (signé J. Prat) }
$$

Ego Joannes Prat guiolensis apud Ruthenos duodecimum profiteor apud acta universitatis medicina monspeliensis die 2 mensis maii anni 1731

$$
\text { (signé J. Prat) }
$$

Grâce à Monsieur Yves Laissus, nous reproduisons ci-dessous la copie exactement faite d'une lettre ${ }^{21}$ de Jean Prat à Jussieu. ${ }^{22}$ Jean Prat fut médecin du roi et naturaliste à la Nouvelle-Orléans, de 1735 à 1746.

\section{Monsieur,}

A La Nelle Orléans, le 29e Juin 1740.

Je comptois d'envoyer ma lettre cy jointe du $2^{\text {e }}$ juin par un navire mart qui devoit partir il y a trois semaines, mais il a voulu attendre le vaysseau du roi. J'adiouteray icy que j'ay embarqué, après beaucoup de sollicitations, une quaisse contenant deux pieds d'une $n^{\text {1le }}$ espèce de phlomis, dix pieds d'une $n^{\text {1le }}$ espèce d'alisier, dix asiminiers et six pieds d'un arbuste appelé arbre à quatre épices; le fruit de cet arbrisseau est une baye ovale qui noircit en meurissant, et qui a un gout approchant de celuy des quatre épices, les sauvages et les pauvres habitants s'en servent pour assaisonner leurs ragouts; je n'ay pas eu occasion de voir la fleur de ces arbrisseaux.

Je vous envoye une autre lettre d'échange cy incluse sur $\mathrm{M}^{\mathrm{rs}}$ les thrésoriers gñaux de la marine de $2000 \mathrm{~L}$ payables en avril 1741 , de laquelle il devroit revenir à mon frère $1400 \mathrm{~L}$ mais comme il me redoit $404 \mathrm{~L} 9 \mathrm{~S}$ suivant le compte cy joint, reste a onze cents cinq livres onze sols, que vous aurez la bonté de luy compter lorsque vous serez payé de cette dernière lettre d'échange.

\footnotetext{
${ }^{20}$ Registre des inscriptions... commencé le $1^{\text {er }}$ janvier 1725 et fini le XI may 1734. Archives de l'Université de Montpellier.

21 Muséum National d'Histoire naturelle, Laboratoire de Phanérogamie, liasse de documents numérotés de 1 à 62, correspondance Prat-Jussieu, no 40 .

22 Bernard de Jussieu, né en 1699, pensionnaire botaniste depuis 1739, mort en 1777.
} 
J'ay tiré une lettre d'échange sur vous de $800 \mathrm{~L}$ payables à vüe à M. Rateau. Quand vous aurez receu mes appointements de 1739 et de $\mathbf{1 7 4 0}$, et le montant des deux lettres d'échange vous aurez à moy $7722 \mathrm{~L} 9 \mathrm{~S}$ dont je destine $6000 \mathrm{~L}$ pour payer l'habitation de mon frère, et pour ce qui restera je vous prie de remettre cinq ou six cents livres à $\mathrm{Mr}$ Molin mart droguiste (Je suppose que vous ne luy avez encore rien remis) pour être employées à exécuter le mémoire des remèdes que je luy ay envoyé, en retranchant la moitié de chaque article, et le restant à Mr votre frère le mart qui l'employera suivant ce que je luy indique. Je vous envoye mon certificat de vie, et celui du thrésorier de la colonie. Si mon frère acquiesce à ma proposition pour le prix de son habitation, il faut qu'il vous envoye une double quittance tant en son nom qu'à celui de sa femme. Vous aurez la bonté de m'en envoyer une et vous garderez l'autre; mais s'il n'acceptoit point mon offre vous pouvez remettre à $\mathrm{Mr}$ votre frère le marchand trois ou quatre mille livres ou davantage s'il souhaite les employer de société avec ses fonds.

Je joins icy un etat de plantes usüelles dont nous avons besoin, Je prie Mgr le comte de Maurepas de s'adresser à vous pour nous les procurer; je vous en auray en mon particulier beaucoup d'obligation. Que d'embarras et de peine ne vous donnay-je pas ? Pourquoy m'inspirez-vous tant de confiance par les bontez que vous ne cessez point de me faire éprouver ? Vous vous trouveriez bien récompensé si vous receviez d'icy une abondante moisson, et je n'ai pû jusqu'à présent vous marquer que ma bonne volonté. Débarrassez moy, s'il est possible, d'une partie du soin de l'hopital, et vous verrez bientôt les effects de ma bonne intention. J'écris à Monsieur de Buffon pour le prier de se joindre à vous afin de m'obtenir quelques facilitez. Quelles espérances ne m'avoit-on pas données, pour le logement, le fret, l'entretien d'un jardin et le Brevet de médecin du port que j'avois demandé ? Je n'ay encore rien vu que des promesses stériles. Je suis bien faché que vous vous soyez donné tant de peine inutilement pour l'amour de moy. J'auray bientôt passé icy six années sans vous avoir satisfait en ce que vous aviez lieu d'attendre de moy (faute de secours nécessaires) accablé d'ailleurs de fatigues, et de maladies, par des soins qu'il faut que je donne à un hopital dépourvu presque de tout secours, à quoy je suis nécessairement obligé de suppléer. Si je suis peu satisfait de ce coté là, je le suis encore moins du coté de ma petite fortune, qui se trouvera réduite à ce que vous aurez entre vos mains à la fin de cette année. Recevez je vous prie, Monsieur, ces plaintes de la part d'une personne qui a toute la confiance dans l'un de ses meilleurs amys. 
Voicy un tas de pieces que mon frère m'a demandées pour faire tenir à son procureur, si vous le connoissez vous pourrez les luy remettre, sinon vous attendrez que mon frère vous le fasse connoitre. Je demande au ministre la permission de faire embarquer deux laboureurs de mon pays, par le premier vaysseau, mon frère doit me les envoyer, si leur passage est accordé vous me ferez playsir de luy en donner avis, sans quoy il ne pourroit pas risquer de les faire partir.

Je vous envoye dans une petite boëtte des racines du polygala à fleur blanche que j'ay trouvé cette année, une espèce de graines qui m'ont paru singulières et que j'ay receues de cent lieues d'icy de la part d'un homme à qui j'avois fait présent de 24 pots d'eau de vie qui coute icy $4 \mathrm{~L}$ le pot, jugez si les gens de ce pays ne sont pas bien reconnoissants; vous trouverez aussy un morceau de bitume que les sauvages Atacapa ramassent sur le bord de la mer, il m'a paru avoir du rapport à l'asphaltum.

Fol. 2

Vous me ferez playsir de m'apprendre les effects que vous ou Monsieur votre frère aurez remarqué de l'usage du polygala. Je l'employerai aussi a la premiere occasion. Je finis en vous assurant que je suis avec les sentiments de l'attachement le plus parfait et d'une entiere reconnoissance

\section{Monsieur}

Votre tres humble et tres obeissant serviteur

\section{Prat}

Monsieur de Jussieu trouvera icy de nouvelles assurances de mon tres humble respect. $\mathrm{M}^{\mathrm{r}}$ de vandermonde, et de la serre, de la continuation de mon amitié.

La correspondance Prat-Jussieu est conservée, sous forme de microfilm et de photocopie, au Département d'Histoire de l'Université de Montréal; elle a fait l'objet d'un compte rendu dont le texte a été communiqué à Monsieur Antoine Roy, Archiviste de la province de Québec.

\section{ROLAND LAMONTAGNE}

Université de Montréal. 